Article

\title{
Enantioselective Pharmacokinetics of $\alpha$-Lipoic Acid in Rats
}

\section{Ryota Uchida ${ }^{1}$, Hinako Okamoto ${ }^{2,3}$, Naoko Ikuta ${ }^{3}$, Keiji Terao ${ }^{2,3}$ and Takashi Hirota ${ }^{1, *}$}

1 Department of Biopharmaceutics, Faculty of Pharmaceutical Science, Tokyo University of Science, 2641 Yamazaki, Noda-shi, Chiba 278-8510, Japan; E-Mail: j3b13702@ed.tus.ac.jp

2 CycloChem Bio Co., Ltd., KIBC654R 5-5-2 Minatojima-minamimachi, Chuo-ku, Kobe 650-0047, Japan; E-Mails: hinako.okamoto@cyclochem.com (H.O.); keiji.terao@cyclochem.com (K.T.)

3 Graduate School of Medicine, Kobe University, 7-5-2 Kusunoki-cho, Chuo-ku, Kobe 650-0017, Japan; E-Mail: naoko.ikuta@people.kobe-u.ac.jp

* Author to whom correspondence should be addressed; E-Mail: hirotas5@rs.noda.tus.ac.jp; Tel./Fax: +81-4-7121-4450.

Academic Editor: Mateus Webba da Silva

Received: 20 August 2015 / Accepted: 11 September 2015 / Published: 21 September 2015

\begin{abstract}
Lipoic acid (LA) is widely used for nutritional supplements as a racemic mixture, even though the $\mathrm{R}$ enantiomer is biologically active. After oral administration of the racemic mixture ( $R$ - $\alpha$-lipoic acid (RLA) and $S$ - $\alpha$-lipoic acid (SLA) mixed at the ratio of 50:50) to rats, RLA showed higher plasma concentration than SLA, and its area under the plasma concentration-time curve from time zero to the last $(A U C)$ was significantly about 1.26 times higher than that of SLA. However, after intravenous administration of the racemic mixture, the pharmacokinetic profiles, initial concentration $\left(C_{0}\right), A U C$, and half-life $\left(T_{1 / 2}\right)$ of the enantiomers were not significantly different. After oral and intraduodenal administration of the racemic mixture to pyrolus-ligated rats, the $A U C$ s of RLA were significantly about 1.24 and 1.32 times higher than that of SLA, respectively. In addition, after intraportal administration the AUC of RLA was significantly 1.16 times higher than that of SLA. In conclusion, the enantioselective pharmacokinetics of LA in rats arose from the fraction absorbed multiplied by gastrointestinal availability $\left(F_{a} F_{g}\right)$ and hepatic availability $\left(F_{h}\right)$, and not from the total clearance.
\end{abstract}

Keywords: $\alpha$-lipoic acid; pharmacokinetics; enantioselective; gastrointestinal availability; hepatic availability; clearance; rat 


\section{Introduction}

In the 1930s, $\alpha$-lipoic acid (LA; 5-(1,2-dithiolan-3-yl) pentanoic acid) was found to be a growth factor of bacteria [1] and was first isolated from the bovine liver in 1950 [2]. In the 1960s, LA had already started to be used for patients with liver cirrhosis [3] or mushroom poisoning [4]. In addition, a number of studies have addressed the character and efficacy of LA, and those results were reviewed by several researchers [5-7]. LA has been attracting a great deal of attention because of its multiple functions. LA has two sulfur atoms, one each at the $\mathrm{C} 6$ and $\mathrm{C} 8$ carbons, in its molecule. They are connected by a disulfide bond, and because the C6 carbon is chiral, LA exists as two enantiomers ( $R$ - and $S$-forms of LA, Figure 1).
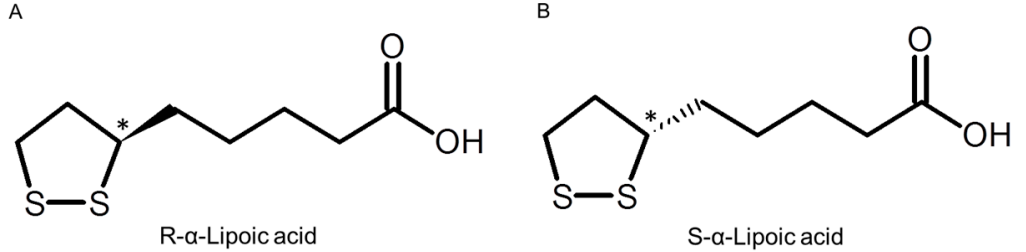

Figure 1. Structure of $R$ - $\alpha$-lipoic acid (A) and $S$ - $\alpha$-lipoic acid (B). Chiral center shown with asterisk $(*)$.

$R$ - $\alpha$-lipoic acid (RLA) is biosynthesized from octanoic acid in mitochondria [8-11]. It is a natural form of LA [12], and works as a cofactor of various mitochondrial respiratory chain enzymes such as pyruvate, $\alpha$-ketoglutarate, and branched-chain $\alpha$-ketoacid dehydrogenases [13]. Both forms of LA seem to have different potencies. The $R$-form is more potent than the $S$-form in its ability to stimulate glucose uptake in L6 myotubes, as well as to increase insulin-stimulated glucose uptake in obese Zucker rats [14]. Endogenously-synthesized LA is covalently bound to specific proteins, which function as cofactors for mitochondrial dehydrogenase enzyme complexes [15]. In addition, to the physiological functions of protein bound LA, there is an increasing scientific and medical interest in potential therapeutic uses of pharmacological doses of free LA [16]. LA's antioxidant properties consist of the following: (1) its capacity to scavenge reactive oxygen species (ROS) directly; (2) its ability to regenerate endogenous antioxidants, such as glutathione and vitamins $\mathrm{E}$ and $\mathrm{C}$; and (3) its metal-chelating activity, resulting in reduced ROS production. Moreover, LA plays a pivotal role as antioxidant and metabolic component of some enzymatic complexes involved in glucose metabolism of different cell types [17]. Due largely to its antioxidant properties, LA has recently been reported to afford protection against oxidative injury in various disease processes, including neurodegenerative disorders [7]. Streeper et al. [18] suggested that RLA enhances insulin-stimulated glucose transport and glucose metabolism in insulin-resistant rat skeletal muscle more than SLA. Hagen et al. [19] suggested that RLA-supplemented aged rats had improved mitochondrial function. On the other hand, $S$ - $\alpha$-lipoic acid (SLA) was reported not to function as a cofactor of respiratory chain enzymes [20,21]. Moreover, Gal reported that SLA was more lethal than RLA in thiamine-deficient rats [22]. Wessel et al. [23] also reported that SLA caused higher mortality in streptozotocin-induced diabetic rats compared to RLA. Thus, RLA would be preferred to racemic LA ( $r a c-L A)$ as a drug or nutritional supplement. However, because of the lower melting point of RLA $\left(46-49^{\circ} \mathrm{C}\right)$ than that of $\mathrm{rac}$-LA $\left(60-62^{\circ} \mathrm{C}\right)$, it is difficult and costly to press into tablets. Hence, most of the LA formulations are supplied as its racemic form in consideration of its stability and ease of manufacturing. 
On the other hand, several reports indeed demonstrated enantioselective pharmacokinetics of LA. Hermann et al. and Niebch et al. [24,25] suggested SLA was cleared more rapidly than RLA after infusion of rac-LA to humans. In addition, several groups reported the enantioselective absorption of LA after oral administration of rac-LA [24,26-29]. However, little is known about the mechanisms of enantioselective pharmacokinetics in detail.

In the present study, we aimed to clarify the mechanism of enantioselective pharmacokinetics of LA. Thus, we compared the systemic exposures after administration of the racemic mixture to rats by several routes and the stability of both enantiomers in different $\mathrm{pH}$ solutions based on the gastrointestinal conditions.

\section{Results}

\subsection{Chiral Separation of LA ( $\alpha$-Lipoic Acid)}

Typical chromatograms for the analysis of SLA and RLA are shown in Figure 2. The peaks with retention times of 4.95 and $5.56 \mathrm{~min}$ were assigned to SLA and RLA, respectively. The peak resolution calculated from the chromatograms was over 1.5. Meanwhile, the peaks with retention times of 4.92 and 5.53 min were assigned to SLA-d5 and RLA-d5 (internal standards), respectively, and its value of resolution was also over 1.5. The lower limit of quantification for each enantiomer was $5 \mathrm{ng} / \mathrm{mL}$ (Figure 2B). The range of the calibration curve was $5-1250 \mathrm{ng} / \mathrm{mL}$, and the chromatogram of a sample at the upper limit is shown in Figure 2C. The linearity of each calibration curve was excellent $(r>0.999)$. No ghost peaks were observed (Figure 2A).

A
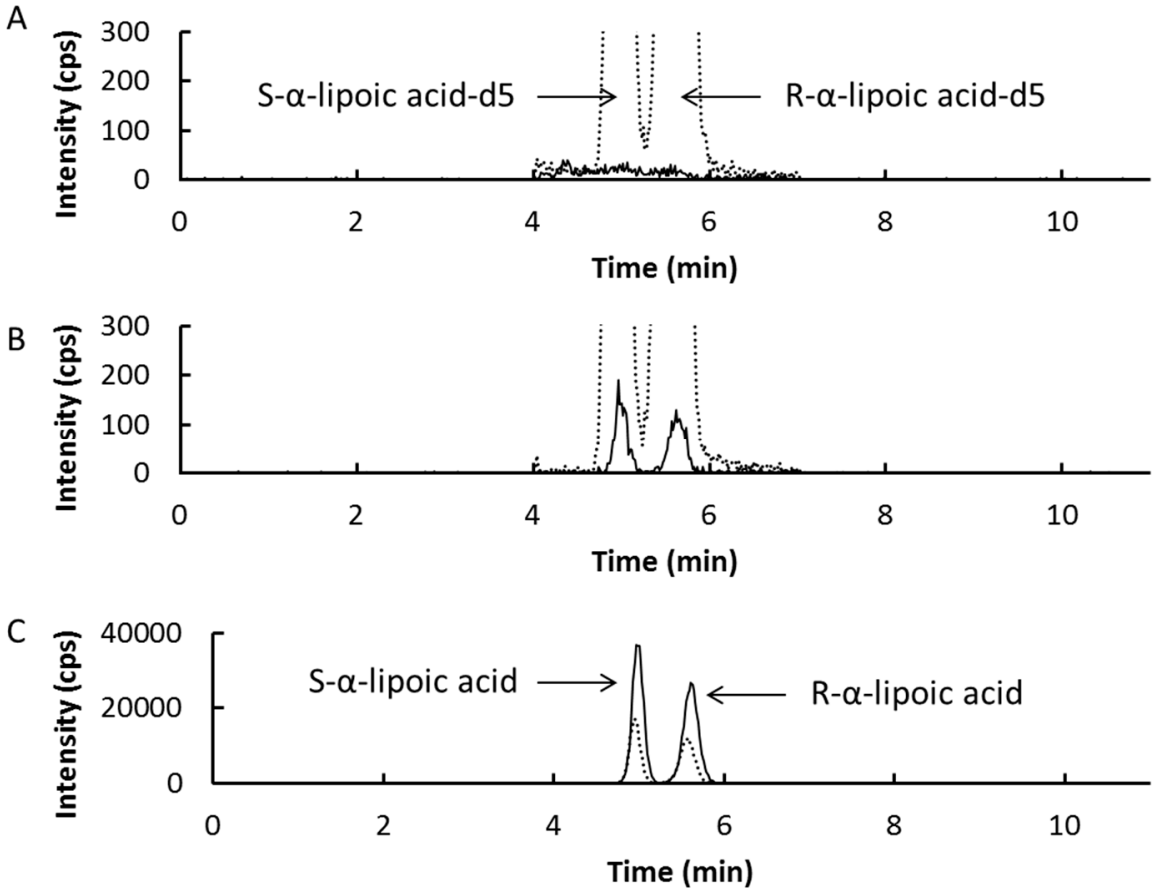

Figure 2. Representative chromatograms for $\alpha$-lipoic acid chiral separation in plasma. Chromatograms shown with solid and dotted line are for $\alpha$-lipoic acid and $\alpha$-lipoic acid-d5 (internal standard), respectively. Blank plasma (A); blank plasma spiked with $\alpha$-lipoic acid ( $5 \mathrm{ng}$ of each enantiomer/mL, B); and blank plasma spiked with $\alpha$-lipoic acid (1250 $\mathrm{ng}$ of each enantiomer/mL, C). 


\subsection{Stability in Different $p H$}

The stability test of each enantiomer was performed in various $\mathrm{pH}$ solutions. After adding the racemic mixture to the solutions at a concentration of $10 \mathrm{mg} \mathrm{LA} / \mathrm{mL}$, each residual concentration in the solution was measured for $60 \mathrm{~min}$. No significant difference in residual ratio was observed between the enantiomers in any of the solutions (Table 1). The solutions at $\mathrm{pH}$ of 1.2 and 3 were simulated gastric fluid of humans and rats [30-32], respectively, and that at $\mathrm{pH}$ of 6.8 was simulated small intestinal fluid.

Table 1. The stability of $\alpha$-lipoic acid in various $\mathrm{pH}$ solutions.

\begin{tabular}{ccccccc}
\hline & \multicolumn{9}{c}{ Residual Rate (\%) } \\
\cline { 2 - 7 } Time (min) & \multicolumn{2}{c}{ pH 1.2 } & \multicolumn{2}{c}{ pH 3.0 } & \multicolumn{2}{c}{ pH 6.8 } \\
\cline { 2 - 7 } & RLA & SLA & RLA & SLA & RLA & SLA \\
\hline 0 & 100 & 100 & 100 & 100 & 100 & 100 \\
1 & $22.8 \pm 3.7$ & $22.6 \pm 3.7$ & $95.5 \pm 1.0$ & $95.4 \pm 0.4$ & $100.2 \pm 2.1$ & $100.0 \pm 1.9$ \\
5 & $17.4 \pm 2.9$ & $17.4 \pm 2.8$ & $98.4 \pm 2.0$ & $97.8 \pm 0.9$ & $99.5 \pm 0.4$ & $99.4 \pm 0.3$ \\
15 & $14.5 \pm 3.5$ & $14.3 \pm 3.4$ & $97.2 \pm 0.7$ & $98.1 \pm 1.1$ & $101.7 \pm 1.8$ & $101.4 \pm 1.8$ \\
30 & $12.7 \pm 1.2$ & $12.7 \pm 1.2$ & $99.1 \pm 3.2$ & $99.2 \pm 3.4$ & $98.9 \pm 1.3$ & $99.2 \pm 1.4$ \\
60 & $11.8 \pm 0.7$ & $11.7 \pm 0.9$ & $99.9 \pm 1.4$ & $99.7 \pm 1.2$ & $99.6 \pm 1.1$ & $99.8 \pm 0.9$ \\
\hline
\end{tabular}

Residual rate are shown as mean \pm standard deviation $(n=3)$. RLA, $R$ - $\alpha$-lipoic acid; SLA, $S$ - $\alpha$-lipoic acid. Statistical analysis was performed by using the paired- $t$ test at each time point of each $\mathrm{pH}$ condition.

\subsection{Pharmacokinetic Profiles}

\subsubsection{Oral and Intravenous Administration}

Plasma concentrations of LA were measured after oral administration of the racemic mixture (20 mg LA/kg, $2 \mathrm{~mL} / \mathrm{kg}$, Figure 3A) or intravenous administration ( $5 \mathrm{mg} \mathrm{LA} / \mathrm{kg}, 1 \mathrm{~mL} / \mathrm{kg}$, Figure 3B) of the racemic mixture to rats. Although the time to peak $\left(T_{\max }\right)$ was not significantly different between the enantiomers, the maximum plasma concentration $\left(C_{\max }\right)$ and area under the plasma concentration versus time curve from time zero to the last $(A U C)$ of RLA after oral administration were significantly about 1.26 times higher than those of SLA $(p<0.01$, Figure 3A, Table 2). On the other hand, after intravenous administration, the plasma concentration profiles and their pharmacokinetic parameters the initial plasma concentration $\left(C_{0}\right)$, half-life $\left(T_{1 / 2}\right)$ and $A U C$ were not significant different between the enantiomers (Figure 3B, Table 2).

A

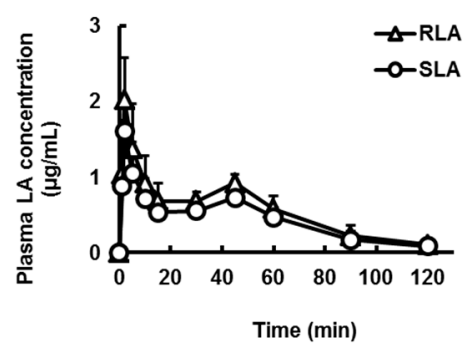

B

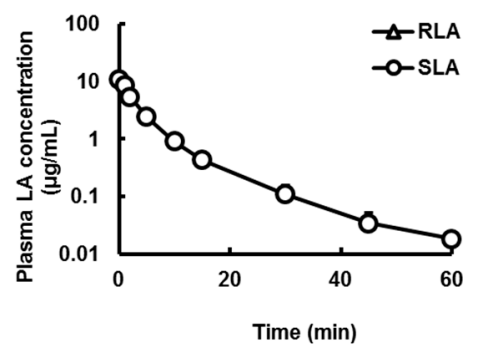

Figure 3. Plasma concentration-time profiles of $\alpha$-lipoic acid after oral (A) and intravenous (B) administration of the racemic mixture. Data are shown as mean \pm standard deviation $(n=4)$. 
Table 2. Pharmacokinetic parameters of $\alpha$-lipoic acid after oral and intravenous administration of the racemic mixture to rats.

\begin{tabular}{ccccc}
\hline Pharmacokinetic & \multicolumn{2}{c}{ Oral } & \multicolumn{2}{c}{ Intravenous } \\
\cline { 2 - 5 } Parameters & RLA & SLA & RLA & SLA \\
\hline$C_{\max }$ or $C_{0}(\mu \mathrm{g} / \mathrm{mL})$ & $2.0 \pm 0.6$ & $1.6 \pm 0.4 *$ & $11.4 \pm 3.6$ & $10.9 \pm 3.2$ \\
$T_{\max }(\min )$ & $2.0 \pm 0.0$ & $2.0 \pm 0.0$ & not determined & not determined \\
$T_{1 / 2}(\mathrm{~min})$ & $26.7 \pm 7.1$ & $26.9 \pm 7.1$ & $10.9 \pm 1.1$ & $12.2 \pm 2.3$ \\
$A U C(\mu \mathrm{g} \cdot \mathrm{min} / \mathrm{mL})$ & $67.7 \pm 6.8$ & $53.8 \pm 5.2 *$ & $48.2 \pm 3.4$ & $46.0 \pm 2.3$ \\
\hline
\end{tabular}

Pharmacokinetic parameters are shown as mean \pm deviation standard $(n=4)$. RLA, $R$ - $\alpha$-lipoic acid; SLA, $S$ - $\alpha$-lipoic acid; $C_{\max }$, maximum plasma concentration; $C_{0}$, initial concentration; $T_{\max }$, time of maximum plasma concentration; $T_{1 / 2}$, half-life; $A U C$, area under the plasma concentration versus time curve from time 0 to the last; *, probability $(p)<0.01$ compared with RLA. Statistical analysis was performed by using the paired- $t$ test.

\subsubsection{Absorption from Stomach and Small Intestine}

Plasma LA concentrations were measured after oral and intraduodenal administration of the racemic mixture (20 mg LA/kg, $2 \mathrm{~mL} / \mathrm{kg}$, Figure 4) to rats with pylorus ligation. In the case of this study, the pharmacokinetics profiles of oral administration were represented as the absorption from the stomach, because the stomach was completely separated from the intestine. As was the case with the oral administration mentioned above, although the $T_{\max }$ was the same between the two enantiomers, the $C_{\max }$ and $A U C$ of RLA after oral administration were significantly about 1.16 and 1.24 times higher than those of SLA, respectively ( $p<0.01$, Figure 4A, Table 3 ). On the other hand, after intraduodenal administration, $T_{\max }$ was not significantly different between the enantiomers. Furthermore, the $C_{\max }$ and $A U C$ of RLA after intraduodenal administration were significantly about 1.28 and 1.32 times higher than SLA, respectively ( $p<0.01$, Figure 4B, Table 3 ). However, the $C_{\max }$ and $A U C$ after intraduodenal administration were several times higher than those after oral administration.

A

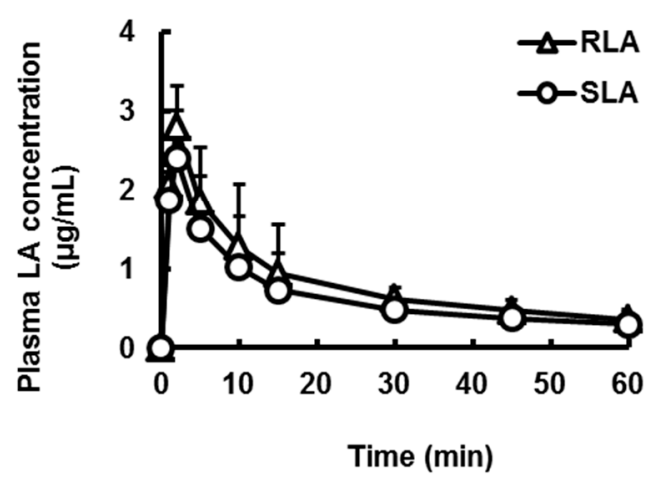

B

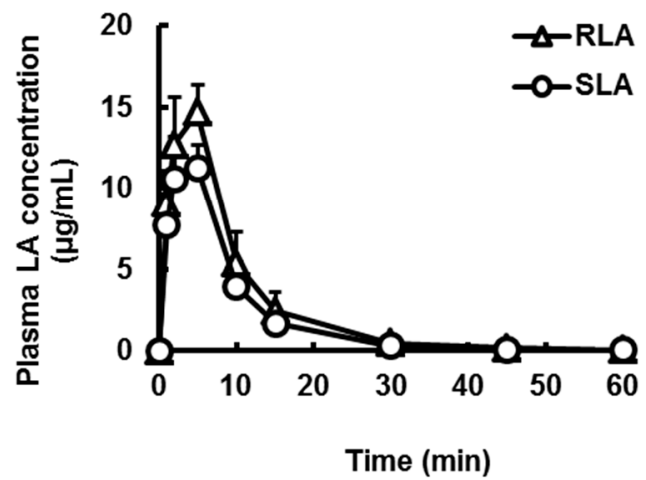

Figure 4. Plasma concentration-time profiles of $\alpha$-lipoic acid after oral (A) and intraduodenal (B) administration of the racemic mixture to pylorus ligated rats. Data are shown as mean \pm standard deviation $(n=4)$. 
Table 3. Pharmacokinetic parameters of $\alpha$-lipoic acid after oral and intraduodenal administration of the racemic mixture to pylorus ligated rats.

\begin{tabular}{ccccc}
\hline Pharmacokinetic & \multicolumn{2}{c}{ Oral } & \multicolumn{2}{c}{ Intraduodenal } \\
\cline { 2 - 5 } Parameters & RLA & SLA & RLA & SLA \\
\hline$C_{\max }(\mu \mathrm{g} / \mathrm{mL})$ & $2.8 \pm 0.5$ & $2.4 \pm 0.6^{*}$ & $14.7 \pm 1.7$ & $11.5 \pm 1.7 *$ \\
$T_{\max }(\mathrm{min})$ & $2.0 \pm 0.0$ & $2.0 \pm 0.0$ & $5.0 \pm 0.0$ & $4.3 \pm 1.5$ \\
$A U C(\mu \mathrm{g} \cdot \mathrm{min} / \mathrm{mL})$ & $48.1 \pm 15.6$ & $38.8 \pm 13.2 *$ & $154.2 \pm 11.3$ & $116.5 \pm 4.4 *$ \\
\hline
\end{tabular}

Pharmacokinetic parameters are shown as mean \pm standard deviation $(n=4)$. RLA, $R$ - $\alpha$-lipoic acid; SLA, $S$ - $\alpha$-lipoic acid; $C_{\max }$, maximum plasma concentration; $T_{\max }$, time of maximum plasma concentration; $A U C$, area under the plasma concentration versus time curve from time 0 to the last; *, probability $(p)<0.01$ compared with RLA. Statistical analysis was performed by using the paired- $t$ test.

\subsubsection{Hepatic Availability}

To clarify the enantioselectivity in hepatic availability, rats were administered the racemic mixture of LA via the portal vein $(5 \mathrm{mg} \mathrm{LA} / \mathrm{kg}, 1 \mathrm{~mL} / \mathrm{kg})$ to rats. The plasma LA concentrations are shown in Figure 5 . The $A U C$ of RLA was significantly about 1.16 times higher than that of SLA (Figure 5, Table 4).

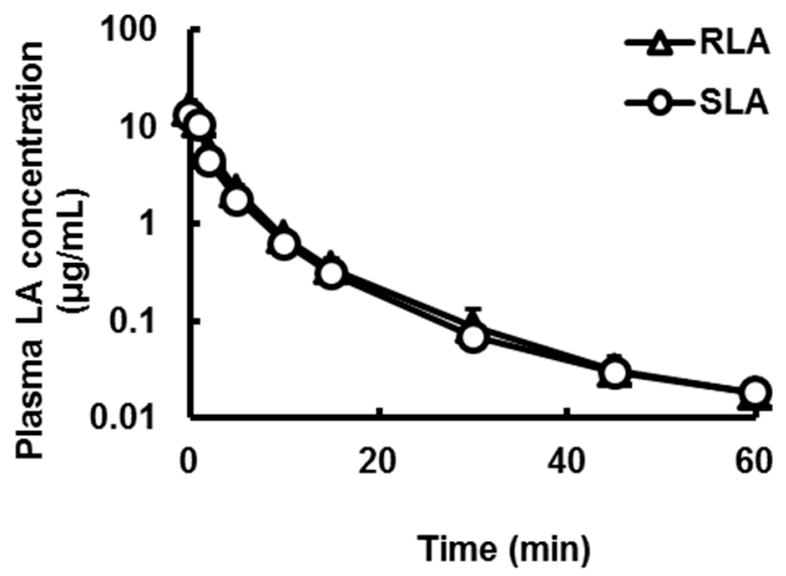

Figure 5. Plasma concentration-time profiles of $\alpha$-lipoic acid after intraportal administration of the racemic mixture of LA to rats. Data are shown as mean \pm standard deviation $(n=4)$.

Table 4. Pharmacokinetic parameters of $\alpha$-lipoic acid after intraportal administration of the racemic mixture of LA to rats.

\begin{tabular}{ccc}
\hline Pharmacokinetic Parameters & RLA & SLA \\
\hline$C_{0}(\mu \mathrm{g} / \mathrm{mL})$ & $14.7 \pm 3.9$ & $13.2 \pm 2.7$ \\
$T_{1 / 2}(\mathrm{~min})$ & $13.9 \pm 0.6$ & $15.7 \pm 1.0$ \\
$A U C(\mu \mathrm{g} \cdot \mathrm{min} / \mathrm{mL})$ & $47.5 \pm 6.1$ & $41.0 \pm 5.1 *$ \\
\hline
\end{tabular}

Pharmacokinetic parameters are shown as mean \pm standard deviation $(n=4)$. RLA, $R$ - $\alpha$-lipoic acid; SLA, $S$ - $\alpha$-lipoic acid; $C_{0}$, initial plasma concentration; $T_{1 / 2}$, half-life; $A U C$, area under the plasma concentration versus time curve (from initial to last points); *, probability $(p)<0.01$ compared with RLA. Statistical analysis was performed by the paired- $t$ test. 


\section{Discussion}

In the drug development of a chiral molecule, when one of them is a eutomer (pharmacologically effective) and the other is a distomer (biologically inactive or toxic), and the enantiomers are not easily isomerized in the body, the eutomer should be provided as much as possible (FDA guideline in 1992, [33]). However, in the case of many nutritional supplements, racemic mixtures are still provided without sufficient verification of each enantiomer because the asymmetric synthesis and chiral separation are costly. Most LA formulations for supplements and drugs in the market are also racemic mixtures. Maddux et al. [34] reported each enantiomer was equally effective at protecting against oxidative stress-induced insulin resistance. On the other hand, Streeper et al. and Hagen et al. [18,19] reported RLA was effective in several symptoms. Indeed, the enantioselective pharmacological effects of LA might be unclear. Nevertheless, optically pure RLA was recommended in the view of safety since SLA was reported to be the distomer [22,23]. Furthermore, although several researchers suggested RLA was superior to SLA with respect to the enantioselective pharmacokinetics [24-29], the detailed mechanisms have not been revealed so far. In the present study, we examined the causes of the enantioselective pharmacokinetics.

In advance of the pharmacokinetic experiments, an assay method of LA enantiomers was developed in order to determine plasma concentrations efficiently. Previously, although the separation method was reported by Niebch et al. [25], it was complicated with derivatization steps and was time-consuming. Hence, we developed a simple and rapid method with reference to Kobayashi et al. [35] as described in the Result section. It was appropriate for the present pharmacokinetics study.

After oral administration of the racemic mixture of LA to rats, although the values of $T_{\max }$ were not different, the $C_{\max }$ and $A U C$ of RLA were 1.26 times higher than those of SLA (Figure 3A and Table 2). Gleiter et al. [26] also reported the enantioselective pharmacokinetic profiles of LA after oral administration of a racemic mixture in humans. According to their and our results, it was found that the enantioselective pharmacokinetics in humans and rats tended to be qualitatively similar.

To clarify the mechanism of the enantioselective pharmacokinetics, the racemic mixture was administered intravenously to rats. The plasma concentration profiles and their pharmacokinetic parameters were not different between the enantiomers (Figure 3B and Table 2). Hence there was no difference in clearance between RLA and SLA. This result differed from the report of Hermann et al. and Niebch et al. [24,25], which suggested that the clearance of SLA was higher than that of RLA in humans. One of the causes of this discrepancy might be just a species difference, because the metabolic pathway of LA was proposed to be different between rats and humans [36]. From our results, at least in rats, we considered that the enantioselective pharmacokinetics after oral administration arose from the selectivity in a transporting process to the systemic circulation, probably that of absorption, or the stability in the gastrointestinal tract, and not from a difference of total clearance. Meanwhile the plasma $\mathrm{T}_{1 / 2}$ in the present study with rats was shorter than that in human studies [24,27,28]. This result was consistent with the common brief that the smaller animals were, the higher clearance they had [37].

In vitro stability of LA was evaluated against various $\mathrm{pH}$ conditions which simulated the gastrointestinal fluid. The pKa of LA is 4.7 [38], and Ikuta et al. [39] reported that RLA is aggregated under acidic conditions ( $\mathrm{pH}$ 1.2). However, no difference in residual ratio was observed between RLA and SLA at $\mathrm{pH} 3.0$, which was reported to be the gastric $\mathrm{pH}$ of fasted rats [30,31] or at $\mathrm{pH} 6.8$ (Table 1). Even at pH 1.2 in simulated gastric juice, no difference was observed between the enantiomers even 
though some of the samples were precipitated just after mixing (Table 1). These results suggested that the cause of enantioselective pharmacokinetics did not occur before absorption. In this regard, however, it is difficult to definitively conclude that it happens after absorption. RLA and SLA were reported to have different modes of binding to proteins [40] and, thus, further experiments should be conducted in vitro in the appropriate conditions not only of $\mathrm{pH}$ but also the presence of some digestive enzymes and proteins to simulate actual gastrointestinal fluid.

Then, we examined where the enantioselective absorption occurred in the gastrointestinal tract. We previously reported that RLA was rapidly absorbed from the stomach to some extent [41]. Based on this, we evaluated the absorption separately from the stomach and small intestine after administration of the racemic mixture to pylorus ligated rats. The $A U C$ of RLA after oral and intraduodenal administration was about 1.24 and 1.32 times higher than that of SLA, respectively (Figure 3 and Table 3), even though the $C_{\max }$ and $A U C$ of each enantiomer following the small intestinal route were several times higher than those following the gastric route. These results showed that the main contribution to the enantioselective pharmacokinetics of LA arises from absorption from the small intestine.

In general, oral bioavailability $(F)$ is defined as a product of the fraction absorbed $\left(F_{a}\right)$, gastrointestinal availability $\left(F_{g}\right)$, and hepatic availability $\left(F_{h}\right)$. Thus, intraportal administration of the racemic mixture was performed to clarify whether the fraction absorbed multiplied by gastrointestinal availability $\left(F_{a} F_{g}\right)$ or $F_{h}$ contributed to the enantioselective absorption observed. The $A U C$ of RLA was significantly higher than that of SLA. However, the difference was smaller than the difference in $A U C$ ratio of RLA/SLA after oral administration (1.26 times). This result suggested that the enantioselectivity arose from the difference not only of $F_{h}$ but also of the $F_{a} F_{g}$ in the transfer from the intestinal tract to systemic circulation. Takaishi et al. clearly indicated that the absorption of LA was mediated by transporters such as a monocarboxylate transporter and a sodium-dependent multivitamin transporter [42]. However, it is unclear whether the transporters contribute to the enantioselective transport because they did not assay individual enantiomers. Hence, future study using cultured cell lines will be needed to clarify whether this transport is enantioselective.

Meanwhile, the degree of enantioselectivity in rats observed in this study was not so large, that is, the RLA/SLA ratio in $A U C$ is only 1.25. Gleiter et al. [26], reported that the difference was about 1.85 times in humans. As mentioned before, Hermann et al. and Niebch et al. indicated the clearance of SLA was faster than that of RLA in humans [24,25]. Thus, the enantioselective pharmacokinetics in humans might be caused by the differences not only in the transfer from the intestinal tract to systemic circulation but also after the transfer to systemic circulation.

\section{Experimental Section}

\subsection{Chemical and Reagents}

RLA-Na (purity $>98.0 \%$ ) and SLA-Na (purity $>85.0 \%$ ) were purchased from Changshu Fushilai Medicine and Chemical Co., Ltd. (Changshu, China). Rac-LA (purity $>98.0 \%$ ) was purchased from Sigma-Aldrich Production GmbH (Buchs, Switzerland). Rac-LA-d5 (purity $>98.0 \%$ ) was purchased from Toronto Research Chemicals Inc. (Toronto, ON, Canada). All other chemicals and reagents were commercially available and of analytical grade or higher. 


\subsection{Animals}

Male Sprague-Dawley (SD) rats were obtained from Japan SLC Inc. (Hamamatsu, Japan), and used at the age of eight weeks (230-270 g) after at least one week of acclimatization. All rats were housed in a temperature $-\left(23 \pm 1{ }^{\circ} \mathrm{C}\right)$ and humidity $-(55 \% \pm 5 \%)$ controlled room with $12 \mathrm{~h} \mathrm{light/dark}$ cycle. Water and food (Labo MR Stock, Nosan Corporation, Yokohama, Japan) were available ad libitum throughout the study except as described below. The rats were fasted for at least $12 \mathrm{~h}$ before drug administration and drugs were administered under isoflurane anesthesia regardless of the administration route. After the experiments, the rats were killed by exsanguination also under anesthesia. All rats were handled in accordance with the institutional and national guidelines for the care and use of laboratory animals.

\subsection{In Vitro Stability Test}

The simulated gastric and intestinal fluids were prepared at $\mathrm{pH} 1.2,3.0$ and 6.8. Five hundred $\mu \mathrm{L}$ of the simulated gastric fluid or intestinal fluid was added into $1.5 \mathrm{~mL}$ tubes, and the tubes were warmed at $37{ }^{\circ} \mathrm{C}$ in water bath for $30 \mathrm{~min}$. After preincubation, $500 \mu \mathrm{L}$ of racemic LA solution $(10 \mathrm{mg} \mathrm{LA} / \mathrm{mL})$ dissolved in $0.5 \%(w / v)$ carboxymethylcellulose sodium salt $(\mathrm{CMC}-\mathrm{Na})$ was added into the tubes. Aliquots of the samples $(50 \mu \mathrm{L})$ of the mixture were withdrawn at $1,5,15,30$, and 60 min under $37^{\circ} \mathrm{C}$. As a reference (time 0 ), water warmed at $37{ }^{\circ} \mathrm{C}$ was used instead of the simulated fluids. The collected solutions were filtered with a $0.22 \mu \mathrm{m}$ centrifugal filter device (Ultrafree ${ }^{\circledR}$-MC, Millipore, Bedford, MA, USA) by centrifuging at $4000 \times g$ for $15 \mathrm{~s}$. Then, the filtrates were serially diluted 2500 -fold with water, and stored at $-20{ }^{\circ} \mathrm{C}$ until the analysis. Residual ratio was calculated by following equation.

$$
\text { Residual ratio }(\%)=\frac{\text { peak area ratio } \text { each time point }_{\text {time }}}{\text { peak } \text { area ratio }_{\text {time }}} \times 100
$$

\subsection{Drug Administration}

\subsubsection{Oral and Intravenous Administration}

RLA-Na and SLA-Na dissolved in CMC-Na were mixed at the ratio of 50:50 (racemic mixture), and this racemic mixture was orally administered to rats $(20 \mathrm{mg} \mathrm{LA} / \mathrm{kg}, 2 \mathrm{~mL} / \mathrm{kg}, n=4)$ by a feeding tube. The dose was determined by reference to clinical studies [43]. The racemic mixture dissolved in saline was intravenously administered to rats $(5 \mathrm{mg} \mathrm{LA} / \mathrm{kg}, 1 \mathrm{~mL} / \mathrm{kg}, n=4)$ via the caudal vein.

\subsubsection{Oral Administration under Pylorus Ligation}

The procedure of surgery and administration was performed as previously reported [41]. Briefly, under isoflurane anesthesia, the abdomen was opened, the pylorus was slightly lifted and ligated with cotton thread, and then the incision was closed immediately with suture and an adhesive was applied. After surgery, racemic mixture was orally administered to the rats $(20 \mathrm{mg} \mathrm{LA} / \mathrm{kg}, 2 \mathrm{~mL} / \mathrm{kg}, n=4)$. The absorption from the stomach was evaluated based on the results of this experiment. 


\subsubsection{Intraduodenal Administration}

Just before pylorus ligation in the course of the operation described above, racemic mixture was injected from a syringe with a 22-gauge needle into the duodenum of the rats through the gastric corpus (20 mg LA/kg, $2 \mathrm{~mL} / \mathrm{kg}, n=4$ ). Immediately after the injection, the pylorus was tightly ligated with suture to prevent reflux of the compound back into the stomach. The incision was closed with suture and adhesive was applied.

\subsubsection{Intraportal Administration}

Under isoflurane anesthesia, the abdomen was opened and racemic mixture dissolved in saline was injected from a syringe with a 30 -gauge needle into the hepatic portal vein $(5 \mathrm{mg} \mathrm{LA} / \mathrm{kg}, 1 \mathrm{~mL} / \mathrm{kg}$, $n=4)$. Immediately after the injection, the puncture was covered with adhesive and the incision was closed with suture and adhesive was applied.

\subsection{Blood Collection}

Blood was withdrawn from the external jugular vein using heparinized syringes under isoflurane anesthesia at 0 (predose), 1, 2, 5, 10, 15, 30, 45, 60, 90, and $120 \mathrm{~min}$ or at the same time points until $60 \mathrm{~min}$ after drug administration. The collected blood was centrifuged at $3000 \times \mathrm{g}$ and $4{ }^{\circ} \mathrm{C}$ for $10 \mathrm{~min}$ to obtain plasma. Plasma was stored at $-20{ }^{\circ} \mathrm{C}$ until the analysis.

\subsection{Determination of LA Concentration by LC-MS/MS}

The LC-MS/MS system consisted of API 3200 with a Shimadzu Prominence HPLC system (Shimadzu, Kyoto, Japan). The HPLC system consisted of a LC-20AD binary pump, DGU-20A3 degasser, SIL-20A autosampler, CTO-20A column oven, and CBM-20A system controller. The measurement was performed using the method of Kobayashi et al. [35] with modification. Briefly, $200 \mu \mathrm{L}$ of acetonitrile containing $0.1 \%(v / v)$ formic acid and $200 \mathrm{ng} / \mathrm{mL}$ of rac-LA-d5 as an internal standard was added to a $50 \mu \mathrm{L}$ sample. After mixing, the sample was centrifuged at $10,800 \times g$ and $4{ }^{\circ} \mathrm{C}$ for $10 \mathrm{~min}$. Ten $\mu \mathrm{L}$ of the supernatant was applied onto the LC-MS/MS system. The HPLC was fitted with a CHIRALPAK AD-RH column $(5 \mu \mathrm{m}, 2.1 \times 150 \mathrm{~mm}$, Daicel, Osaka, Japan), and chromatography was performed using a gradient elution program at a flow rate of $0.3 \mathrm{~mL} / \mathrm{min}$. The column temperature was maintained at $30^{\circ} \mathrm{C}$. The mobile phase consisted of $0.1 \%(v / v)$ formic acid/water (A) and $0.1 \%(v / v)$ formic acid/methanol (B). The gradient program was as follows: start the ratio of $\mathrm{A} / \mathrm{B}$ at $60 / 40$, increase $\mathrm{B}$ linearly from $40 \%$ to $95 \%$ between $t=0$ and $1.0 \mathrm{~min}$, then hold the ratio of $\mathrm{A} / \mathrm{B}$ at $5 / 95$ until $t=6.0 \mathrm{~min}$, decrease $\mathrm{B}$ linearly from $95 \%$ to $40 \%$ between $t=6.0$ and $6.1 \mathrm{~min}$, then hold the ratio of $\mathrm{A} / \mathrm{B}$ at $60 / 40$ until $t=11.0 \mathrm{~min}$. The analytes and internal standard from the column were detected by the negative ion mode, and analyzed by multiple reaction monitoring mode of the transitions $\mathrm{m} / \mathrm{z} 205.0$ to 170.8 for $\mathrm{rac}$-LA and $\mathrm{m} / \mathrm{z} 210.0$ to 173.8 for $\mathrm{rac}$-LA-d5. 


\subsection{Pharmacokinetics Analysis}

The $C_{\max }$ and $T_{\max }$ were obtained directly from the individual plasma concentration-time profiles. The $C_{0}$ and $T_{1 / 2}$ were calculated using the macro program MOMENT (EXCEL) [44]. The first and last three points of the logarithmic plasma concentration were used for linear regression of $C_{0}$ and $T_{1 / 2}$, respectively. The $A U C$ of LA was estimated using the trapezoidal rule.

\subsection{Statistical Analysis}

Parameters are presented as arithmetic mean \pm standard deviation. The pharmacokinetic parameters were compared with paired- $t$ tests for comparison of each enantiomer. Differences were considered statistically significant when $p<0.01$.

\section{Conclusions}

We revealed the following findings about enantioselective pharmacokinetics of LA. (1) The exposure to RLA is higher than that to SLA; (2) The enantioselectivity occurred in the absorption phase, and not in the elimination phase. The $F_{h}$ and $F_{a}$ and/or $F_{g}$ in a transfer process from the gastrointestinal tract, mainly the small intestine, to systemic circulation are implicated in the enantioselective pharmacokinetics. According to these results and the observation that RLA is a eutomer, a formulation of the single enantiomer RLA would be more suitable for oral administration of LA than that of a racemic mixture.

\section{Acknowledgments}

We would like to thank Donald Hinman for scientific advice and editing the manuscript.

\section{Author Contributions}

Ryota Uchida and Takashi Hirota contributed to the conception and design of the study; Ryota Uchida contributed to the experiences of the tested samples; Ryota Uchida and Takashi Hirota contributed to the drafting of the manuscript; Ryota Uchida, Hinako Okamoto, Naoko Ikuta, Keiji Terao and Takashi Hirota approved the final version of the manuscript.

\section{Conflicts of Interest}

The authors declare no conflict of interest.

\section{References}

1. Snell, E.E.; Strong, F.M.; Peterson, W.H. Growth factors for bacteria: Fractionation and properties of an accessory factor for lactic acid bacteria. Biochem. J. 1937, 31, 1789-1799.

2. Reed, L.J.; De, B.B.; Gunsalus, I.C.; Hornberger, C.S. Crystalline $\alpha$-lipoic acid; a catalytic agent associated with pyruvate dehydrogenase. Science 1951, 114, 93-94.

3. Bravo, M.; Orrego-Matte, H.; Walshe, J.M. Carbohydrate intermediates in hepatic cirrhosis: Role of lipoic acid. J. Lab. Clin. Med. 1961, 57, 213-222. 
4. Zulik, R.; Bako, F.; Budavari, J. Death-cap poisoning. Lancet 1972, 2, doi:10.1016/S01 40-6736(72)91659-5

5. Packer, L.; Witt, E.H.; Tritschler, H.J. $\alpha$-Lipoic acid as a biological antioxidant. Free Radic. Biol. Med. 1995, 19, 227-250.

6. Biewenga, G.P.; Haenen, G.R.; Bast, A. The pharmacology of the antioxidant lipoic acid. Gen. Pharmacol. 1997, 29, 315-331.

7. Evans, J.L.; Goldfine, I.D. $\alpha$-Lipoic acid: A multifunctional antioxidant that improves insulin sensitivity in patients with type 2 diabetes. Diabetes Technol. Ther. 2000, 2, 401-413.

8. Parry, R.J. Biosynthesis of lipoic acid. 1. Incorporation of specifically tritiated octanoic acid into lipoic acid. J. Am. Chem. Soc. 1977, 99, 6464-6466.

9. Parry, R.J.; Trainor, D.A. Biosynthesis of lipoic acid. 2. Stereochemistry of sulfur introduction at C-6 of octanoic acid. J. Am. Chem. Soc. 1978, 100, 5243-5244.

10. Wada, H.; Shintani, D.; Ohlrogge, J. Why do mitochondria synthesize fatty acids? Evidence for involvement in lipoic acid production. Proc. Natl. Acad. Sci. USA 1997, 94, 1591-1596.

11. Morikawa, T.; Yasuno, R.; Wada, H. Do mammalian cells synthesize lipoic acid?: Identification of a mouse cDNA encoding a lipoic acid synthase located in mitochondria. FEBS Lett. 2001, 498, 16-21.

12. Brookes, M.H.; Golding, B.T.; Howes, D.A.; Hudson, A.T. Proof that the absolute configuration of natural $\alpha$-lipoic acid is $R$ by the synthesis of its enantiomer $((S)-(-)-\alpha$-lipoic acid) from $(S)$-malic acid. J. Chem. Soc. Chem. Commun. 1983, 19, 1051-1053.

13. Reed, L.J. From lipoic acid to multi-enzyme complexes. Protein Sci. 1998, 7, 220-224.

14. Khanna, S.; Roy, S.; Packer, L.; Sen, C.K. Cytokine-induced glucose uptake in skeletal muscle: Redox regulation and the role of $\alpha$-lipoic acid. Am. J. Physiol 1999, 276, R1327-R1333.

15. Bramanti, V.; Tomassoni, D.; Bronzi, D.; Grasso, S.; Curro, M.; Avitabile, M.; Volsi, G.L.; Renis, M.; Ientile, R.; Amenta, F.; et al. $\alpha$-Lipoic acid modulates GFAP, vimentin, nestin, cyclin $\mathrm{D}_{1}$ and MAP-kinase expression in astroglial cell cultures. Neurochem. Res. 2010, 35, 2070-2077.

16. Krämer, K.; Packer, L. R- $\alpha$-lipoic acid. In Nutraceuticals in Health and Disease Prevention; Krämer, K., Hoppe , P.P., Packer, L., Eds.; CRC Press: Boca Raton, FL, USA, 2001; pp. 129-164.

17. Grasso, S.; Bramanti, V.; Tomassoni, D.; Bronzi, D.; Malfa, G.; Traini, E.; Napoli, M.; Renis, M.; Amenta, F.; Avola, R. Effect of lipoic acid and $\alpha$-glyceryl-phosphoryl-choline on astroglial cell proliferation and differentiation in primary culture. J. Neurosci. Res. 2014, 92, 86-94.

18. Streeper, R.S.; Henriksen, E.J.; Jacob, S.; Hokama, J.Y.; Fogt, D.L.; Tritschler, H.J. Differential effects of lipoic acid stereoisomers on glucose metabolism in insulin-resistant skeletal muscle. Am. J. Physiol. 1997, 273, E185-E191.

19. Hagen, T.M.; Ingersoll, R.T.; Lykkesfeldt, J.; Liu, J.; Wehr, C.M.; Vinarsky, V.; Bartholomew, J.C.; Ames, A.B. $(R)-\alpha$-Lipoic acid-supplemented old rats have improved mitochondrial function, decreased oxidative damage, and increased metabolic rate. FASEB J. 1999, 13, 411-418.

20. Yang, Y.S.; Frey, P.A. 2-Ketoacid dehydrogenase complexes of Escherichia coli: Stereospecificities of the three components for (R)-lipoate. Arch. Biochem. Biophys. 1989, 268, 465-474.

21. Oehring, R.; Bisswanger, H. Incorporation of the enantiomers of lipoic acid into the pyruvate dehydrogenase complex from Escherichia coli in vivo. Biol. Chem. Hoppe Seyler 1992, 373, 333-335.

22. Gal, E.M. Reversal of selective toxicity of (-)- $\alpha$-lipoic acid by thiamine in thiamine-deficient rats. Nature 1965, 207, doi:10.1038/207535a0. 
23. Wessel, K.; Borbe, H.; Ulrich, H.; Hettche, H.; Bisswanger, H.; Packer, L.; Klip, A. Use of $R$-(+)- $\alpha$-lipoic acid, $R$-(-)-dihydrolipoic acid and metabolites in the form of the free acid or as salts or esters or amides for the preparation of drugs for the treatment of diabetes mellitus as well as of its sequelae. U.S. Patent 6284787 B1, 4 September 2001.

24. Hermann, R.; Niebch, G.; Borbe, H.O.; Fieger-Büschges, H.; Ruus, P.; Nowak, H.; Riethmüller-Winzen, H.; Peukert, M.; Blume, H. Enantioselective pharmacokinetics and bioavailability of different racemic $\alpha$-lipoic acid formulations in healthy volunteers. Eur. J. Pharm. Sci. 1996, 4, $167-174$.

25. Niebch, G.; Buchele, B.; Blome, J.; Grieb, S.; Brandt, G.; Kampa, P.; Raffel, H.H.; Locher, M.; Borbe, H.O.; Nubert, I.; et al. Enantioselective high-performance liquid chromatography assay of $(+) \mathrm{R}-$ and (-)S- $\alpha$-lipoic acid in human plasma. Chirality 1997, 9, 32-36.

26. Gleiter, C.H.; Schug, B.S.; Hermann, R.; Elze, M.; Blume, H.H.; Gundert-Remy, U. Influence of food intake on the bioavailability of thioctic acid enantiomers. Eur. J. Clin. Pharmacol. 1996, 50, 513-514.

27. Hermann, R.; Wildgrube, H.J.; Ruus, P.; Niebch, G.; Nowak, H.; Gleiter, C.H. Gastric emptying in patients with insulin dependent diabetes mellitus and bioavailability of thioctic acid-enantiomers. Eur. J. Pharm. Sci. 1998, 6, 27-37.

28. Breithaupt-Grogler, K.; Niebch, G.; Schneider, E.; Erb, K.; Hermann, R.; Blume, H.H.; Schug, B.S.; Belz, G.G. Dose-proportionality of oral thioctic acid--coincidence of assessments via pooled plasma and individual data. Eur J. Pharm. Sci. 1999, 8, 57-65.

29. Hermann, R.; Mungo, J.; Cnota, P.J.; Ziegler, D. Enantiomer-selective pharmacokinetics, oral bioavailability, and sex effects of various $\alpha$-lipoic acid dosage forms. Clin. Pharmacol. 2014, 6, 195-204.

30. McConnell, E.L.; Basit, A.W.; Murdan, S. Measurements of rat and mouse gastrointestinal pH, fluid and lymphoid tissue, and implications for in vivo experiments. J. Pharm. Pharmacol. 2008, 60, 63-70.

31. Ward, F.W.; Coates, M.E. Gastrointestinal pH measurement in rats: Influence of the microbial flora, diet and fasting. Lab. Anim. 1987, 21, 216-222.

32. Lui, C.Y.; Amidon, G.L.; Berardi, R.R.; Fleisher, D.; Youngberg, C.; Dressman, J.B. Comparison of gastrointestinal $\mathrm{pH}$ in dogs and humans: Implications on the use of the beagle dog as a model for oral absorption in humans. J. Pharm. Sci. 1986, 75, 271-274.

33. FDA's policy statement for the development of new stereoisomeric drugs. Chirality 1992, 4, 338-340.

34. Maddux, B.A.; See, W.; Lawrence, J.C., Jr.; Goldfine, A.L.; Goldfine, I.D.; Evans, J.L. Protection against oxidative stress-induced insulin resistance in rat L6 muscle cells by mircomolar concentrations of alpha-lipoic acid. Diabetes 2001, 50, 404-410.

35. Kobayashi, Y.; Saito, K.; Iwasaki, Y.; Ito, R.; Nakazawa, H. Enantiomeric determination of $\alpha$-lipoic acid in dietary supplements by liquid chromatography/mass spectrometry. Bunseki Kagaku 2012, 61, 109-114.

36. Schupke, H.; Hempel, R.; Peter, G.; Hermann, R.; Wessel, K.; Engel, J.; Kronbach, T. New metabolic pathways of $\alpha$-lipoic acid. Drug Metab. Dispos. 2001, 29, 855-862.

37. Boxenbaum, H. Interspecies scaling, allometry, physiological time, and the ground plan of pharmacokinetics. J. Pharmacokinet. Biopharm. 1982, 10, 201-227. 
38. Krishnan, C.; Garnett, M. Electrochemical behavior of the super antioxidant, $\alpha$-lipoic acid. Int. J. Electrochem. Sci. 2011, 6, 3607-3630.

39. Ikuta, N.; Sugiyama, H.; Shimosegawa, H.; Nakane, R.; Ishida, Y.; Uekaji, Y.; Nakata, D.; Pallauf, K.; Rimbach, G.; Terao, K.; et al. Analysis of the enhanced stability of R(+)-alpha lipoic acid by the complex formation with cyclodextrins. Int. J. Mol. Sci. 2013, 14, 3639-3655.

40. Suji, G.; Khedkar, S.A.; Singh, S.K.; Kishore, N.; Coutinho, E.C.; Bhor, V.M.; Sivakami, S. Binding of lipoic acid induces conformational change and appearance of a new binding site in methylglyoxal modified serum albumin. Protein J. 2008, 27, 205-214.

41. Uchida, R.; Iwamoto, K.; Nagayama, S.; Miyajima, A.; Okamoto, H.; Ikuta, N.; Fukumi, H.; Terao, K.; Hirota, T. Effect of $\gamma$-cyclodextrin inclusion complex on the absorption of R- $\alpha$-lipoic acid in rats. Int. J. Mol. Sci. 2015, 16, 10105-10120.

42. Takaishi, N.; Yoshida, K.; Satsu, H.; Shimizu, M. Transepithelial transport of $\alpha$-lipoic acid across human intestinal Caco-2 cell monolayers. J. Agric. Food Chem. 2007, 55, 5253-5259.

43. Ziegler, D.; Ametov, A.; Barinov, A.; Dyck, P.J.; Gurieva, I.; Low, P.A.; Munzel, U.; Yakhno, N.; Raz, I.; Novosadova, M.; et al. Oral treatment with $\alpha$-lipoic acid improves symptomatic diabetic polyneuropathy. The sSYDNEY 2 trial. Diabetes Care 2006, 29, 2365-2370.

44. Tabata, K.; Yamaoka, K.; Kaibara, A.; Suzuki, S.; Terakawa, M.; Hata, T. Moment analysis program available on Microsoft Excel ${ }^{\circledR}$. Drug Metab. Pharmacokinet. 1999, 14, 286-293.

(C) 2015 by the authors; licensee MDPI, Basel, Switzerland. This article is an open access article distributed under the terms and conditions of the Creative Commons Attribution license (http://creativecommons.org/licenses/by/4.0/). 